\title{
Dorsal penile nerve block for circumcision in pediatric patients: A prospective, observer-blinded, randomized controlled clinical trial for the comparison of ultrasound-guided vs landmark technique
}

\author{
An Teunkens ${ }^{1,2}$ (I) | Marc Van de Velde ${ }^{1,2}$ | Kristien Vermeulen ${ }^{1}$ | Philippe Van Loon $^{1}$ | \\ Guy Bogaert ${ }^{3}$ | Steffen Fieuws ${ }^{4}$ | Steffen Rex ${ }^{1,2}$
}

${ }^{1}$ Department of Anaesthesiology, University Hospitals of the KU Leuven, Leuven, Belgium

${ }^{2}$ Department of Cardiovascular Sciences, KU Leuven-University of Leuven, Leuven, Belgium

${ }^{3}$ Department of Urology, University Hospitals of the KU Leuven, Leuven, Belgium

${ }^{4}$-Biostat, KU Leuven- University of Leuven, Leuven, Belgium

Correspondence

Dr An Teunkens, Department of

Anaesthesiology, University Hospitals of the KU Leuven, Leuven, Belgium.

Email: An.Teunkens@uzleuven.be

Section Editor: Adrian Bosenberg

\section{Summary}

Background and aims: Circumcision is a frequently performed procedure in day case pediatric surgery. Dorsal penile nerve block has proven its effectiveness for the management of acute postoperative pain after circumcision. We investigated if the ultrasound-guided placement of a dorsal penile nerve block could reduce opioid requirement as compared to a landmark-based technique.

Methods: Three hundred and ten prepubertal children, aged between 52 weeks postconception and 11 years, were included in this prospective, observer-blinded, randomized controlled trial and received either a landmark- or an ultrasound-guided dorsal penile nerve block, using a caudal needle and injecting $0.1 \mathrm{~mL} / \mathrm{kg}$ levobupivacaine $0.5 \%$ bilaterally. A single, experienced investigator performed all blocks. The primary endpoint was the number of patients in need of piritramide postoperatively as triggered by the Objective Pain Scale. Secondary outcome parameters included the cumulative dose of postoperatively administered opioids, the requirement to administer fentanyl intraoperatively, the need for paracetamol and ibuprofen during the first 24 postoperative hours, postoperative pain scores, the incidence of postoperative nausea and vomiting, the anesthesia induction time, and the time to discharge.

Results: The proportion of patients requiring postoperative piritramide did not differ significantly between both groups (Landmark: $38 \%$ vs Ultrasound: $47 \%$, with a difference in proportion between both conditions $(95 \% \mathrm{Cl})$ : $0.09(0.2$ to 0.02$)$; $P=.135)$. In addition, the cumulative doses of postoperative piritramide and intraoperative fentanyl, the postoperative need for paracetamol or ibuprofen, pain scores, the incidence of postoperative nausea and vomiting, and the time to discharge were not different either. However, the anesthesia induction time was significantly longer in the ultrasound-guided dorsal penile nerve block (median time [IQR]: Landmark: 11[9; 13] min vs Ultrasound: 13[11; 15] min, $P<.001$ ).

Conclusion: Compared with the landmark-guided, the ultrasound-guided dorsal penile nerve block did not reduce the need for postoperative analgesia after circumcision in children, but was associated with an increase in the procedural time. 


\section{1 | INTRODUCTION}

Circumcision is a frequently performed procedure in infants and neonates causing significant postoperative pain. ${ }^{1}$ Adequate analgesia is required to minimize acute intra- and postoperative pain and subsequent psychological trauma. ${ }^{2,3}$ Several regional anesthesia techniques have been described for providing effective postoperative analgesia, including caudal block, ring block of the penis, and the dorsal penile nerve block (DPNB). ${ }^{4}$ DPNB has been proven to be an effective, easy, and safe technique., ${ }^{5,6}$ Traditionally, the DPNB has been performed using a landmark-based technique. ${ }^{7}$ In addition, Dalens et al. described the use of a blunt needle with which it is possible to detect the Scarpa layer that is in continuity with Buck's fascia, feeling the pop when slowly progressing through the subcutaneous tissue. Recently, with the increasing use of ultrasound for the placement of peripheral nerve blocks, an ultrasound-guided technique has also been described for DPNB. ${ }^{8,9}$ As a potential advantage, this technique allows us to confirm the spread of the local anesthetic within the sub-pubic space by direct visualization of the Scarpa fascia and might, therefore, increase the success rate of the block. Hence, we hypothesized that in comparison with the landmark-based technique; an ultrasound-guided DPNB would provide better postoperative analgesia and decrease the need for postoperative pain therapy with opioids.

\section{2 | MATERIALS AND METHODS}

\subsection{Study design and population}

In this large prospective, observer-blinded, randomized controlled trial, 310 prepubertal children undergoing elective, radical circumcision in an ambulatory setting were included. The study protocol was approved by the ethics committee of the University Hospitals of the KU Leuven (EC S54289, 25th May 2012), and the Belgian Government (6th June 2012). It was registered in the publicly accessible study register of the European Medicines Agency (EUDRACT 2012-00121716). Patients were enrolled between September 2012 and November 2016. We included 310 children between 3 months (52 weeks postconception) and 11 years old, ASA (American Society of Anaesthesiologists) physical status I-II. Exclusion criteria were an intolerance or allergic reaction against any product used in the study, psychomotor retardation, and the inability to give written informed consent.

Following parental written informed consent, patients were randomly allocated to one of the 2 study groups receiving either a landmark DPNB or an ultrasound DPNB, using a computer-generated random table (Graph Pad Software Inc., La Jolla, CA, USA). Allocation concealment was ensured by enclosing assignments in sealed,

\section{What is already known}

- Circumcision is a frequently performed surgical procedure for which a dorsal penile nerve block has proven its effectiveness. The use of ultrasound increases the success rate of a peripheral nerve block.

\section{What this article adds}

- In our study, using a caudal needle, an ultrasound-guided dorsal penile nerve block technique was compared with the landmark technique in a large pediatric population. We could not demonstrate a decrease in the need for postoperative analgesia while using ultrasound guidance.

opaque, sequentially numbered envelopes, which were brought to the operation room by a study nurse and opened only after the arrival of the patient in the operating theater by the investigator.

\subsection{Study intervention}

Patients in the landmark-group received a DPNB using the Dalens technique with a bilateral injection into the sub-pubic space. ${ }^{7}$ A 22 gauge caudal needle (Epican $\AA$ Paed caudal needle, $45^{\circ}$ Crawford type bevel for epidural anesthesia/analgesia, B. Braun ${ }^{\circledR}$ Medical Inc., Melsungen, Hessen, Germany) was inserted midway between the symphysis pubis and the base of the penis and then advanced until a pop was detected (reflecting the perforation of Scarpa's fascia). After a negative aspiration test, a dose of $0.1 \mathrm{~mL} / \mathrm{kg}$ levobupivacaine 0.5\% (Chirocaine ${ }^{\circledR}$, AbbVie, Wavre, Belgium) was injected on both sides of the penile base. ${ }^{10}$ Patients in the ultrasound-group received a DPNB using the technique described by Sandeman with however a slight modification (Sonosite M-Turbo, linear probe HFL38x, 13$6 \mathrm{MHz}$, Fujifilm Sonosite BV, Amsterdam, The Netherlands). ${ }^{8}$ While in the original technique the ultrasound-probe is placed vertically, we put the probe horizontally between the base of the penis and the symphysis pubis and then adjusted the probe position until the penile shaft and the symphysis pubis were identified (Figure 1). In this ultrasound plane, both sides of the sub-pubic space can be visualized, so that the probe has to be placed only once. Using an out of plane approach, the above-mentioned needle type was inserted into the sub-pubic space under real-time ultrasound guiding, below Scarpa's fascia. $0.1 \mathrm{~mL} / \mathrm{kg}$ levobupivacaine $0.5 \%$ were injected bilaterally under direct ultrasound visualization (Figure 2), to assure spread of the local anesthetic in the sub-pubic space. ${ }^{8}$ In case of an incorrect position of the needle (as visualized by the spread of local anesthetic 


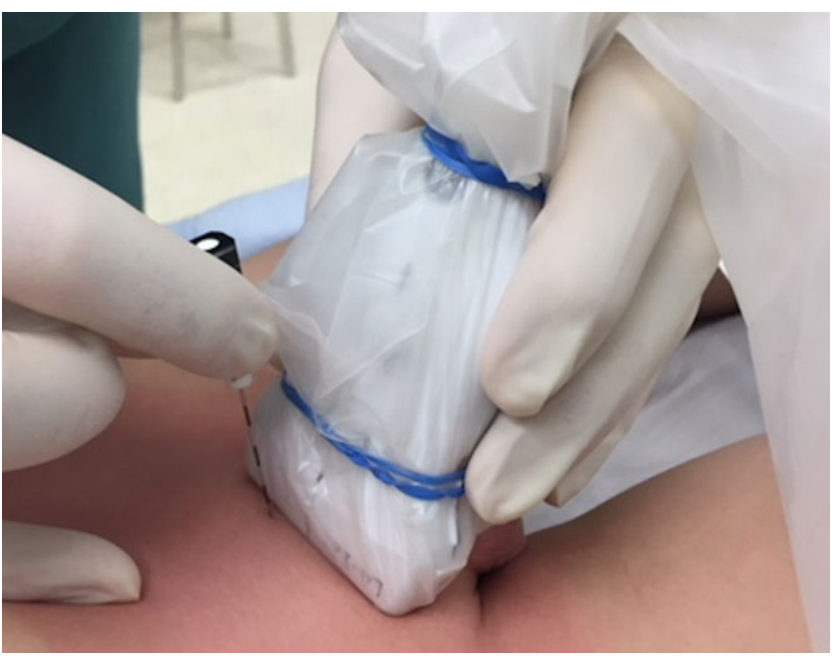

FIGURE 1 Probe and needle positioning

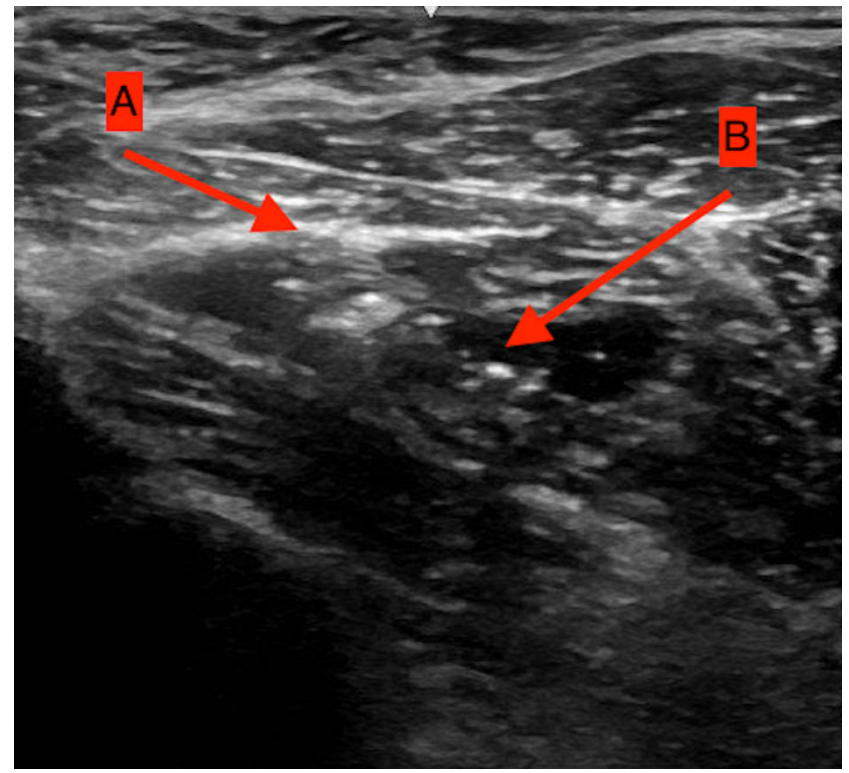

FIGURE 2 Ultrasound image after injection. A, Scarpa's fascia. B, Spread of local anesthetic

above Scarpa's fascia), the needle was re-positioned to ensure a proper position in the sub-pubic space.

All blocks were performed by the same experienced anesthesiologist (AT), who was neither involved in the perioperative care of the patients nor in data gathering and study visits. Anesthesiologists and nurses who were at any time responsible for the follow-up of the study-patients were blinded for the technique used, as were the patients and their parents.

\subsection{Anesthetic and perioperative management}

The anesthetic management was standardized for all patients. Standard monitoring was applied. Anesthesia was induced with inhalation of sevoflurane $8 \%$ in a $50 \% \mathrm{O}_{2} /$ Air mixture. After induction, an IV line was placed, and a pediatric crystalloid infusion was started (combination of saline $0.3 \%$ and glucose $3.3 \%$, Baxter, Deerfield, IL, USA) using the 4-2-1-rule. After an IV bolus of $2 \mathrm{mg} / \mathrm{kg}$ propofol, a laryngeal mask was placed. Subsequently, the DPNB was performed using one of the described techniques. General anesthesia was maintained with sevoflurane $2 \%$ in a $50 \% \mathrm{O}_{2}$ /Air mixture, using pressure controlled ventilation. Spontaneous ventilation was established at the end of surgery.

\section{4 | Perioperative analgesia}

During surgery, opioids were not used routinely. Only when clinical signs of insufficient analgesia were present during surgery (ie, an increase in blood pressure or heart rate $>20 \%$ in comparison to baseline, or patient movements), $1 \mu \mathrm{g} / \mathrm{kg}$ fentanyl was given IV. A standard loading dose of paracetamol $15 \mathrm{mg} / \mathrm{kg}$ was administered after induction of anesthesia.

Postoperatively, the intensity of pain was registered using the Objective Pain Scale. ${ }^{11,12}$ The Objective Pain Scale was scored upon arrival at the post anesthesia care unit and every 10 minutes during the first hour, followed by assessments every 30 mins until discharge and at 24 hours postoperatively at home. Piritramide $0.03 \mathrm{mg} / \mathrm{kg}$ was given if the score was $>3$ and repeated every 10 minutes until pain scores were $\leq 3$. Piritramide is one of the commonly used opioids for the treatment of postoperative pain in our country with a ratio of analgesic potency of 0.7 when compared with morphine. ${ }^{13}$

The analgesic scheme for pain treatment at home consisted of paracetamol suppositories or syrup (dose according to weight class, maximum $15 \mathrm{mg} / \mathrm{kg}$, every 6 hours if needed) and ibuprofen syrup or tablets (dose according to weight class, maximum $10 \mathrm{mg} / \mathrm{kg}$, every 8 hours if required).

\section{5 | Study outcomes}

The primary outcome of the study was the number of patients who needed piritramide IV in the postoperative care unit as triggered by an Objective Pain Scale score $>3$.

Secondary outcome parameters included: cumulative doses of postoperative piritramide, the need to intraoperatively administer fentanyl, the postoperative need for paracetamol and ibuprofen, the Objective Pain Scale score over time, the incidence of postoperative nausea and vomiting, the anesthesia induction time (defined as the time between the start of anesthesia and ready for surgery), and the time to discharge from the hospital.

Moreover, we assessed the safety of both penile nerve block techniques by assessing any adverse events including hematoma, gangrene of the glans due to arterial compression, and signs of local anesthetic systemic toxicity. ${ }^{6}$

\section{3 | STATISTICAL ANALYSIS}

\section{1 | Sample size calculation}

The sample size calculation was performed with SAS, version 9.2 of the SAS system for Windows (SAS Institute Inc., Cary, NC, USA). 
The sample size was calculated to compare the proportion of patients who needed piritramide postoperatively in both groups and was based on a Fisher's exact test (2-sided with alpha $=5 \%$ ). In our center, the landmark-guided technique had been clinical routine for circumcisions already before the start of the trial. A retrospective analysis of 50 patient files showed a postoperative need for piritramide in $10 \%$ of children having received a landmark DPNB. We considered a reduction in the proportion of patients in need for postoperative piritramide from $10 \%$ to $2 \%$ as clinically meaningful. To detect this reduction, 155 patients in each group were needed to achieve $80 \%$ power.

\section{2 | Data analysis}

All statistical analyses were performed using R version 3.3.1 (201606-21) (Team RC. R: A language and environment for statistical computing. R Foundation for statistical computing, Vienna, Austria, 2016).

The Fisher's exact test was used to compare proportions of patients between the LM and US-group. The Mann-Whitney $U$ test was used for variables on the ordinal or ratio level. A linear model for repeated measurements was used to compare the evolution over time for the Objective Pain Scale and vital perioperative parameters. The interaction between measurement moment and condition was tested to verify the evolution over time between both groups.

All tests were performed 2-sided with an alpha $=5 \%$.

For the secondary outcomes measured at different time points, a Bonferroni adjustment was applied to correct for multiple testing.

\section{\begin{tabular}{l|l}
4 & RESULTS
\end{tabular}}

The study flowchart is shown in Figure 3. We enrolled 310 patients who were randomized to receive a DPNB with the landmark technique $(n=155)$ or the ultrasound-guided technique $(n=155)$. All patients underwent the allocated technique. 62 patients were lost to follow-up after 24 hours (Landmark group: 29; Ultrasound group: 33, $P=$.67).

Patients in the 2 groups did not differ concerning demographic data (Table 1).

\section{1 | Primary outcome parameter}

The proportion of patients that needed piritramide postoperatively did not differ significantly between the 2 groups (Landmark: $38 \%$ vs Ultrasound: 47\%; with a difference in proportion between both conditions ( $95 \% \mathrm{Cl}$ ): -0.09 ( -0.2 to 0.02$) ; P=.135$ ).

\subsection{Secondary outcome parameters}

Groups did not differ with respect to the cumulative doses of piritramide administered postoperatively (Landmark: median [IQR] 0.031 [0.029; 0.058] mg/kg vs Ultrasound: 0.03 [0.028; 0.054] mg/ $\mathrm{kg}$, difference in median $(95 \% \mathrm{Cl}): 0(0$ to 0$) ; P=.82)$. Likewise, the proportion of patients who needed fentanyl intraoperatively was not different between both groups (Table 2). In 77\% of these patients, fentanyl was administered upon an increase of heart rate or blood pressure. Neither mean Objective Pain Scale scores (mean difference

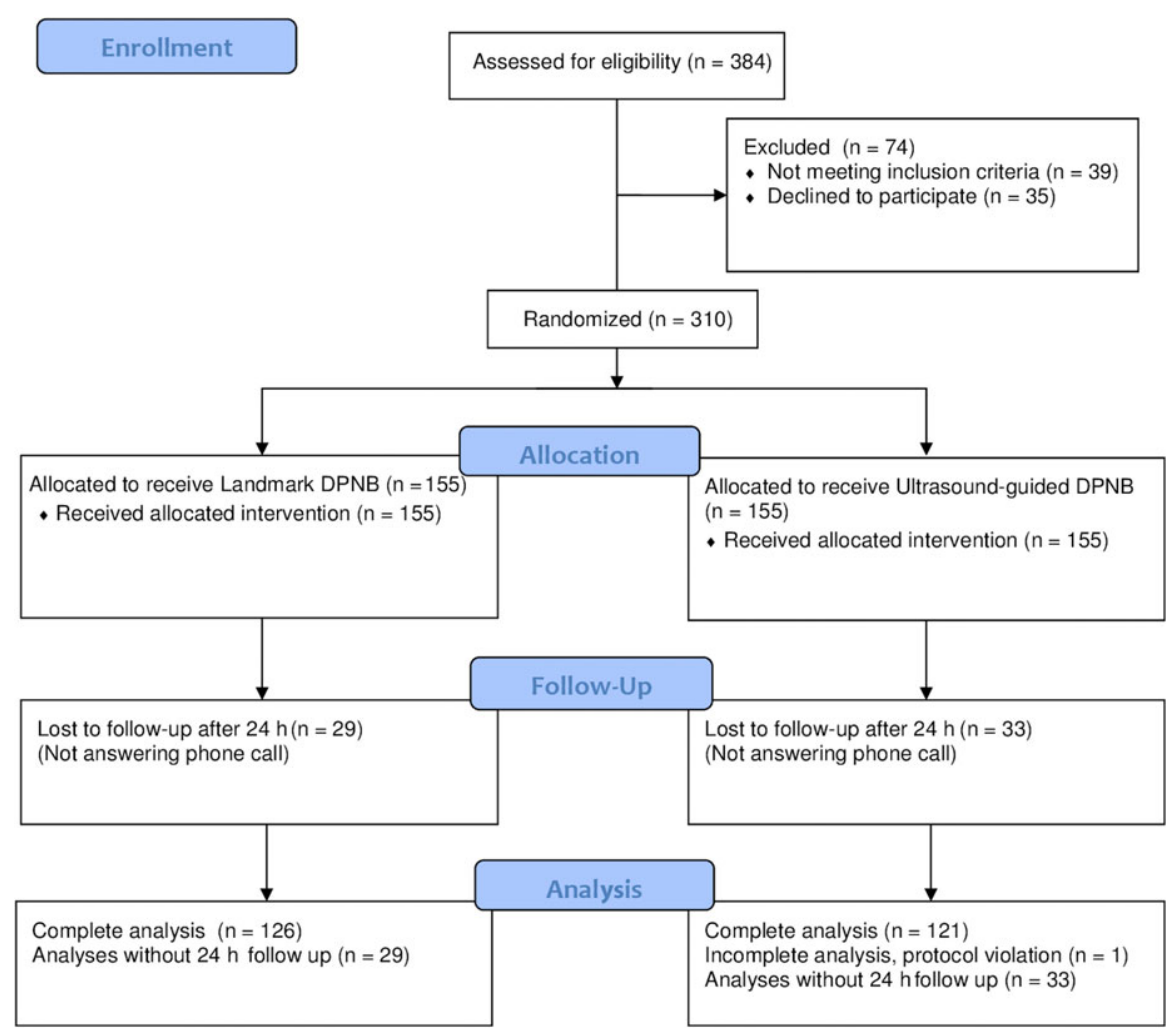

FIGURE 3 Study flowchart ${ }^{26}$ 
TABLE 1 Patient characteristics

\begin{tabular}{llcr} 
& & $\begin{array}{l}\text { Landmark DPNB } \\
\mathbf{n}=155\end{array}$ & \multicolumn{1}{c}{$\begin{array}{l}\text { Ultrasound DPNB } \\
\mathbf{n}=155\end{array}$} \\
\hline Age & Mo & $29[14.5 ; 56.5]$ & $28[15.5 ; 56.5]$ \\
Weight & $\mathrm{Kg}$ & $14[10.7 ; 19.5]$ & $13.3[10.9 ; 18.0]$ \\
\hline ASA & & & \\
\hline ASA 1 & $\mathrm{n}(\%)$ & $150(97)$ & $150(97)$ \\
ASA 2 & $\mathrm{n}(\%)$ & $5(3)$ & $5(3)$ \\
\hline
\end{tabular}

Data are presented as median [IQR] or absolute numbers (with the percentage of the whole).

(95\% Cl): $0.08(-0.16$ to 0.32$) ; P=.506$ ) nor the evolution over time of the Objective Pain Scale scores differed between both groups (Figure 4). No difference was noted between both groups regarding the use of paracetamol or ibuprofen at home (Table 2). There was no difference in the incidence of postoperative nausea and vomiting or the time until discharge but the anesthesia induction time was significantly longer in the ultrasound-guided group (Table 2).

\subsection{Safety outcome}

No adverse events were reported.

\section{5 | DISCUSSION}

The results of this large-scale clinical trial could not demonstrate the superiority of the ultrasound-guided DPNB with respect to postoperative analgesia in pediatric patients undergoing circumcision, thereby refuting our hypothesis that the use of ultrasound in DPNB would improve the success rate of the regional block.

When compared to landmark or nerve stimulator-guided techniques, a superiority of ultrasound-guidance has been demonstrated mainly for blocks that are applied in anatomical regions in which neural structures can be visualized (with peripheral nerve blocks as a paradigm). ${ }^{14,15}$ In truncal blocks, however, ultrasonography is used primarily to identify supra-, inter-, or subfascial spaces rather than to

TABLE 2 Secondary outcome parameters

\begin{tabular}{|c|c|c|c|c|c|c|}
\hline & & Landmark DPNB & Ultrasound DPNB & $\begin{array}{l}\text { Difference in } \\
\text { median }(95 \% \mathrm{Cl})\end{array}$ & $\begin{array}{l}\text { Odds Ratio } \\
(95 \% \mathrm{Cl})\end{array}$ & $P$-value \\
\hline In hospital & & $\mathrm{n}=155$ & $\mathrm{n}=155$ & & & \\
\hline Fentanyl intraoperatively & n (\%) & $41(26)$ & $55(36)$ & & $1.53(0.94-2.49)$ & .11 \\
\hline PONV incidence & n (\%) & $4(3)$ & $3(2)$ & & $0.75(0.17-3.41)$ & 1 \\
\hline Anesthesia induction time & Min & $11[9 ; 13]$ & $13[11 ; 15]$ & $-2(-3$ to -1$)$ & & $<.001$ \\
\hline Discharge time & Min & $179[160 ; 224]$ & $194[168 ; 224]$ & $-7(-17$ to -3$)$ & & .17 \\
\hline At home & & $n=126$ & $n=122$ & & & \\
\hline Paracetamol & n (\%) & $67(53)$ & $71(58)$ & & $1.23(0.74-2.03)$ & .44 \\
\hline Ibuprofen & n (\%) & $59(47)$ & $61(50)$ & & $1.14(0.69-1.87)$ & .70 \\
\hline
\end{tabular}

Data are presented as median [IQR] or absolute number (with the percentage of the whole).

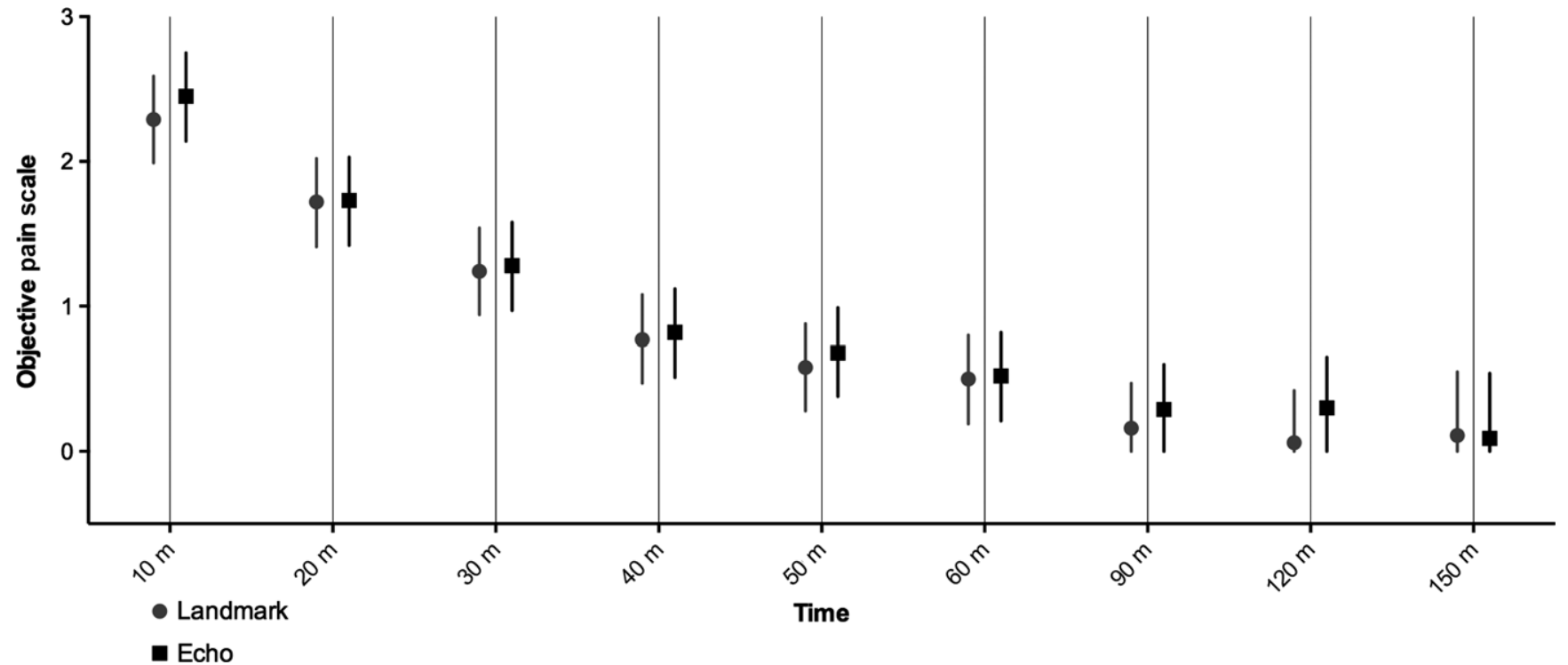

FIG URE 4 Postoperative time course of the Objective Pain Scale (OPS) in both groups. Mean OPS profiles over time for both techniques with $95 \%$ confidence intervals 
directly identify nervous structures. Notably, there is only limited evidence supporting the routine use of ultrasound in truncal blocks. ${ }^{16}$ Also in DPNB, the dorsal penile nerve cannot be directly visualized. Nevertheless, we expected that the proper localization of Scarpa's fascia by ultrasound would improve the accuracy of the needle position and consequently drug deposition. In our study, these potential advantages could however not be translated into a superior efficacy when compared to the landmark DPNB.

Of note, the landmark DPNB relies on the "pop" technique to confirm the correct needle localization. This pop technique is critically dependent upon the operator's experience and skills, and on the design of the needle (with the "pop" being more difficult to detect with a sharp needle). In our study, an experienced anesthesiologist, trained in the landmark technique, performed all DPNB and a blunt caudal needle was used to detect the pop. This study design could have contributed to the fact that we were unable to detect any differences in postoperative analgesia between the 2 techniques and that our findings are in contrast with 2 other clinical trials reporting superiority for ultrasound DPNB. ${ }^{9,17}$ Faraoni and colleagues used a sharp hypodermic needle, and only the same anesthesiologist performed the US-guided blocks. Sandeman and colleagues used a blunt needle, but the blocks were performed either by the principal investigator or an anesthesia trainee under supervision.

Our observations are in correspondence with those of O' Sullivan and colleagues ${ }^{18}$ although they used a sharp hypodermic needle, the block was performed or supervised by an experienced Consultant in pediatric regional anesthesia.

In our study, the anesthesia induction time was significantly longer in the ultrasound group (11 vs 13 minutes), a finding confirmed by Sullivan and colleagues who reported a difference of 75 seconds in advantage of the landmark technique. ${ }^{18}$ However, in our opinion, these differences are likely only of minor clinical relevance. In contrast, in the study of Faraoni and colleagues, ultrasound DPNB was associated with an increase in procedure times of approximately $10 \mathrm{~min}$ when compared with the landmark technique. ${ }^{9}$ Such a difference would clearly limit the use of ultrasound DPNB in a busy day care center.

We acknowledge that our study is subject to several limitations.

First, we did not perform an extra injection of local anesthetic ventrally, at the penile base. Such a ventral injection is necessary to also block the scrotal branches of the pudendal nerve, which provide sensory innervation of the ventral side of the penile skin from the midline to the frenulum in most males. ${ }^{8}$ However, this injection is not part of the "classical" DPNB technique ${ }^{19}$ and is not routinely used in our center. As previous studies have demonstrated its effectiveness in providing complete anesthesia of the penile tip, ${ }^{19,20}$ a comparison of our results with other studies also using the ventral injection is difficult.

Second, it can be discussed whether the Objective Pain Scale for pain assessment is an adequate tool to be used in our population group. The Objective Pain Scale is a behavioral pain scale, appropriate for pain measurement in patients who cannot verbalize their pain ( $<6$ years). For older children, the use of a verbal pain scale is often recommended. ${ }^{12}$ We nevertheless opted for the Objective Pain Scale because it has been validated for all age categories enrolled in our study and given its reliability and ease of use. ${ }^{11,21}$

Third, the Objective Pain Scale scoring system shows some overlap with several scales used to diagnose emergence agitation. Both the Objective Pain Scale and the Paediatric Anesthesia Emergence Delirium scale ${ }^{22}$ consist of items assessing agitation, movements, and inconsolability, owing to the fact that both inadequate pain relief and anesthesia (in particular sevoflurane-anesthesia as used in our study) can cause agitation. ${ }^{23}$ The fact that most children in our study did not receive any opioids intraoperatively even increases their risk to develop emergence agitation. ${ }^{24}$ Discrimination between agitation caused by pain or emergence is often impossible in infants and little children, and hence, treatment of possible pain should be the primary option. ${ }^{23,25}$ In the majority of patients, emergence agitation resolves 15 minutes after end of anesthesia. If agitation continues beyond this time span, it is most probably due to pain, and the requirement of analgesics 15 minutes after extubation should reflect more correctly analgesic potency of the DPNB in our study, eliminating confounding effects of emergence agitation. A subanalysis of piritramide administration in our study showed that piritramide was given in $37 \%$ of patients of the LM-group and $50 \%$ of patients of the US group only during the first 20 minutes after emergence of anesthesia $(P=.16)$, probably treating emergence agitation instead of pain. However, only $24 \%$ of patients of the landmark group and $23 \%$ of patients in the ultrasound group needed piritramide after the first 20 minutes after emergence of anesthesia.

Fourth, the observed percentage of patients in need of postoperative piritramide largely exceeded the observed percentage in the historical group, which was used for the sample size calculation. The difference between the historical cohort and the study population can be most probably attributed to 2 reasons. First, in the historical group, all patients routinely received a standard dose of $1 \mu \mathrm{g} / \mathrm{kg}$ fentanyl intraoperative, while in the trial, opioids were only administered in case of insufficient analgesia, in order to avoid that an unsuccessful block would have been masked by the given fentanyl. Second, in the historical cohort, administration of postoperative analgesia was not based on the Objective Pain Scale, probably facilitating the differentiation of emergence agitation from pain by clinical judgment.

Notably, the difference in the incidence of the primary outcome in the historical cohort and the study population did not reduce the power of our study, since with 155 patients in each group, there would have been still $81 \%$ power to detect a clinically relevant decrease from $40 \%$ to $25 \%$.

Fifth, the study was only powered for the primary outcome parameter. The interpretation of the secondary outcomes should, therefore, be made with caution.

Last, a single operator who was very experienced in the landmark technique performed both the landmark DPNB and the ultrasound DPNB. It cannot be excluded that his experience with the landmark technique also helped him to bring his ultrasound DPNB technique to perfection, and hence led to a performance bias. Moreover, the results of our study do therefore not apply to inexperienced operators. 
Further studies are warranted to assess the impact of the use of ultrasound on the learning curves of "DPNB-novices."

Note that according to our knowledge, the present study is by far the largest ever performed in these setting.

\section{6 | CONCLUSIONS}

In conclusion, the results of this large prospective, randomized, observer-blinded, controlled trial show that the use of an ultrasound-guided DPNB does not decrease the need for postoperative analgesia after circumcision in children compared with the landmark technique when performed by an experienced operator and using a blunt needle.

Parts of this study were presented at ESRA 2017 in Lugano, Switzerland.

\section{ACKOWLEDGMENTS}

Assistance with the study: We express our appreciation for our research nurse Christel Huygens and her team for their valuable assistance.

\section{CONFLICT OF INTEREST}

No conflicts of interest declared.

\section{ORCID}

An Teunkens (iD http://orcid.org/0000-0002-6279-7436

\section{REFERENCES}

1. Bellieni CV, Alagna MG, Buonocore G. Analgesia for infants' circumcision. Ital J Pediatr. BioMed Central; 2013;39:38.

2. Taddio A. Pain management for neonatal circumcision. Paediatr Drugs. 2001;3:101-111.

3. Dixon S, Snyder J, Holve R, Bromberger P. Behavioral effects of circumcision with and without anesthesia. J Dev Behav Pediatr. 1984;5:246-250.

4. Beyaz SG. Comparison of postoperative analgesic efficacy of cauda block versus dorsal penile nerve block with levobupivacaine for circumcision in children. Korean J Pain. 2011:24:31-35.

5. Cyna AM, Middleton P. Caudal epidural block versus other methods of postoperative pain relief for circumcision in boys.In: Cyna AM, ed. Cochrane Database Syst Rev. Chichester, UK: John Wiley \& Sons, Ltd; 2008;37(4): CD003005.

6. Soh CR, Ng SBA, Lim SL. Dorsal penile nerve block. Paediatr Anaesth. 2003:13:329-333.

7. Dalens B, Vanneuville G, Dechelotte P. Penile block via the subpubic space in 100 children. Anesth Analg. 1989;69:41-45.

8. Sandeman DJ, Dilley AV. Ultrasound guided dorsal penile nerve block in children. Anaesth Intensive Care. 2007;35:266-269.

9. Faraoni D, Gilbeau A, Lingier P, Barvais L, Engelman E, Hennart D. Does ultrasound guidance improve the efficacy of dorsal penile nerve block in children? Paediatr Anaesth. Blackwell Publishing Ltd. 2010;20:931-936.
10. Sanford M, Keating GM. Levobupivacaine: a review of its use in regional anaesthesia and pain management. Drugs. Springer International Publishing. 2010;70:761-791.

11. Hannallah RS, Broadman LM, Belman AB, Abramowitz MD, Epstein BS. Comparison of caudal and ilioinguinal/iliohypogastric nerve blocks for control of post-orchiopexy pain in pediatric ambulatory surgery. Anesthesiology. 1987;66:832-834.

12. Beltramini A, Milojevic K, Pateron D. Pain assessment in newborns, infants, and children. Pediatr Ann. SLACK Incorporated 2017;46:e387-e395.

13. Hinrichs M, Weyland A, Bantel C. [Piritramide : A critical review]. Schmerz. 4 ed. Springer Medizin; 2017;31:345-352.

14. Wang $\mathrm{Y}, \mathrm{Wu} \mathrm{T}$, Terry $\mathrm{MJ}$, et al. Improved perioperative analgesia with ultrasound-guided ilioinguinal/iliohypogastric nerve or transversus abdominis plane block for open inguinal surgery: a systematic review and meta-analysis of randomized controlled trials. J Phys Ther Sci. The Society of Physical Therapy Science. 2016;28:1055-1060.

15. Lönnqvist P-A. Is ultrasound guidance mandatory when performing paediatric regional anaesthesia? Curr Opin Anaesthesiol. 2010;23: 337-341.

16. Neal JM, Brull R, Chan VWS, et al. The ASRA evidence-based medicine assessment of ultrasound-guided regional anesthesia and pain medicine: executive summary. Reg Anesth Pain Med 2010;35:S1-S9.

17. Sandeman DJ, Reiner D, Dilley AV, Bennett MH, Kelly KJ. A retrospective audit of three different regional anaesthetic techniques for circumcision in children. Anaesth Intensive Care. 2010;38:519-524.

18. O'Sullivan MJ, Mislovic B, Alexander E. Dorsal penile nerve block for male pediatric circumcision-randomized comparison of ultrasoundguided vs anatomical landmark technique. Pediatr Anesth. 2011;21: 1214-1218.

19. Serour F, Mori J, Barr J. Optimal regional anesthesia for circumcision. Anesth Analg. 1994;79:129-131.

20. Brown TC, Weidner NJ, Bouwmeester J. Dorsal nerve of penis block-anatomical and radiological studies. Anaesth Intensive Care. 1989:17:34-38.

21. Broadman LM, Hannallah RS, Belman AB, Elder PT, Ruttimann $U$, Epstein BS. Post-circumcision analgesia-a prospective evaluation of subcutaneous ring block of the penis. Anesthesiology. 1987;67:399-402.

22. Sikich N, Lerman J. Development and psychometric evaluation of the pediatric anesthesia emergence delirium scale. Anesthesiology. 2004;100:1138-1145.

23. Moore AD, Anghelescu DL. Emergence delirium in pediatric anesthesia. Paediatr Drugs. Springer International Publishing; 2017;19:11-20.

24. Kanaya A. Emergence agitation in children: risk factors, prevention, and treatment. J Anesth. Springer Japan. 2016;30:261-267.

25. Somaini M, Engelhardt T, Fumagalli R, Ingelmo PM. Emergence delirium or pain after anaesthesia-how to distinguish between the two in young children: a retrospective analysis of observational studies. Br J Anaesth. 2016;116:377-383.

26. Moher D, Schulz KF, Altman DG. The CONSORT statement: revised recommendations for improving the quality of reports of parallelgroup randomised trials. The Lancet. 2001;357:1191-1194.

How to cite this article: Teunkens A, Van de Velde M, Vermeulen $\mathrm{K}$, et al. Dorsal penile nerve block for circumcision in pediatric patients: A prospective, observer-blinded, randomized controlled clinical trial for the comparison of ultrasound-guided vs landmark technique. Pediatr Anesth. 2018;28:703-709. https://doi.org/10.1111/pan.13429 